\title{
Elevated Levels of Urinary Markers of Oxidative DNA and RNA Damage in Type 2 Diabetes with Complications
}

\author{
Xinle Liu, ${ }^{1}$ Wei Gan, ${ }^{1,2}$ Yuangao Zou, ${ }^{1}$ Bin Yang, ${ }^{1}$ Zhenzhen Su, ${ }^{1}$ Jin Deng, \\ Lanlan Wang, ${ }^{1}$ and Jianping $\mathrm{Cai}^{2}$ \\ ${ }^{1}$ Department of Lab Medicine, West China Hospital, Sichuan University, Chengdu, Sichuan 610041, China \\ ${ }^{2}$ The Key Laboratory of Geriatrics, Beijing Hospital and Beijing Institute of Geriatrics, Ministry of Health, Beijing 100730, China
}

Correspondence should be addressed to Lanlan Wang; wanglanlan_med@163.com and Jianping Cai; caijp61@vip.sina.com

Received 23 June 2015; Accepted 31 August 2015

Academic Editor: Tim Hofer

Copyright (C) 2016 Xinle Liu et al. This is an open access article distributed under the Creative Commons Attribution License, which permits unrestricted use, distribution, and reproduction in any medium, provided the original work is properly cited.

\begin{abstract}
The mechanisms underlying progression of type 2 diabetes are complex and varied. Recent studies indicated that oxidative stress provided a new sight. To further assess the relationship between nucleic acid oxidation and complications in patients with type 2 diabetes and explore its possible molecular mechanisms, we studied 1316 subjects, including 633 type 2 diabetes patients and 683 age- and sex-matched healthy controls. Urinary levels of DNA oxidation marker 8-oxo-7,8-dihydro-2' ${ }^{\prime}$-deoxyguanosine (8-oxodGuo) and RNA oxidation marker 8-oxo-7,8-dihydroguanosine (8-oxoGuo) were measured by ultraperformance liquid chromatography and mass spectrometry (UPLC-MS/MS). Serum glucose, HbAlc, total cholesterol, HDL cholesterol, LDL cholesterol, and triglycerides (TG) were also determined. The results showed significantly elevated levels of both the urinary 8 oxodGuo and 8-oxoGuo in diabetes patients with/without complications compared with age-matched healthy control subjects ( $p=0.02$ and $p<0.001$, resp.). Patients with complications, especially macrovascular complications, exhibited higher levels of 8 -oxoGuo than those without complications, while there was no difference in the concentrations of serum glucose and lipids. The finding indicates the role for oxidative damage to DNA and RNA, as a molecular mechanism contributing to the progression of type 2 diabetes. Elevated levels of 8-oxoGuo may be a risk factor for type 2 diabetes complications, especially in diabetic macrovascular complications.
\end{abstract}

\section{Introduction}

Type 2 diabetes is a chronic disease characterized by hyperglycemia in the context of insulin resistance and relative lack of insulin. About 382 million people were living with diabetes in 2013 according to the International Diabetes Federation, and by 2035 the number will be almost 600 million [1]. As the prevalence of diabetes has risen to epidemic proportions worldwide, the complications of diabetes have now become one of the most challenging health problems [2]. Thus, new biomarkers that can be used for risk stratification and therapy control as an alternative to current methods are needed.

It has been reported that the occurrence of diabetic complications increased with poor glycaemic control. However, we found that diabetic complications also occurred with better glycaemic control. So there may be other mechanisms for the progression of diabetic complications. Some previous researches on both epidemiology and mechanics suggested that oxidative stress played a pivotal role in the pathogenesis and progression of type 2 diabetes complications [3-7]. And the increased oxidative stress in patients with type 2 diabetes can be caused by hyperglycemia, inflammation, or dyslipidemia [8]. Historically, DNA was the focus of nucleic acid oxidation studies $[9,10]$. However, RNA also comprised an incredibly diverse group of nucleic acids and played vital functions in cellular function and protein synthesis [11]. 8-Oxo-7,8-dihydro-2'-deoxyguanosine (8-oxodGuo) and 8-oxo-7,8-dihydroguanosine (8-oxoGuo) are two main nucleic acid oxidative adducts derived from DNA and RNA, respectively. Many previous studies have shown that levels of urinary 8-oxodGuo were elevated in diabetes patients with complications; however, the evidence on the association 
between urinary 8-oxoGuo and diabetes complications was relatively little $[12,13]$.

In this study, we analyzed the levels of urinary 8-oxodGuo and 8-oxoGuo, serum glucose, and lipids in patients with diabetes and healthy controls. In addition, we investigated the association between 8-oxoGuo and different complications of type 2 diabetes.

\section{Materials and Methods}

2.1. Subjects. The study group consisted of 1,316 subjects, including 633 type 2 diabetes patients and 683 age- and sexmatched healthy controls. All the subjects were recruited from West China Hospital of Sichuan University from January 2014 to October 2014. In the 633 type 2 diabetes patients, 267 patients had complications such as retinopathy, nephropathy, neuropathy, and other vascular complications; the other 366 patients had no complication. All the patients were diagnosed according to the World Health Organization (WHO) diagnostic criteria for type 2 diabetes [14]. Members of the control group, who did not have any personal or family history of either diabetes or dyslipidemia and with normal thyroid, hepatic, and renal functions, were selected from the Health Examination Center of West China Hospital. The study was approved by the Institutional Ethics Committee of West China Hospital of Sichuan University and complied with Declaration of Helsinki. Written informed consent was obtained prior to enrollment from all subjects.

2.2. Measurement of Biochemical Parameters. Blood samples were collected at the fasting state from each subject. Total cholesterol, HDL cholesterol, LDL cholesterol, and triglycerides (TG) were analyzed enzymatically in serum (Modular P800, Roche Diagnostics GmbH, Germany). Glucose was enzymatically determined by using the hexokinase method (Modular P800, Roche Diagnostics GmbH, Germany). HbAlc was determined by a method based on high-performance liquid chromatography (HPLC) which was approved by National Glycohemoglobin Standardization Program (NGSP) (HLC-723 G8, Tosoh Corporation, Japan) in whole blood. Urinary creatinine was analyzed by the Jaffe reaction (Modular P800, Roche Diagnostics GmbH, Germany).

2.3. Measurement of Urinary 8-oxodGuo and 8-oxoGuo. Freshly voided urine samples were obtained from each subject and centrifuged; after that, the supernatants of $1.0 \mathrm{~mL}$ were transferred to Eppendorf tubes and stored at $-80^{\circ} \mathrm{C}$ until they were analyzed. The frozen samples were thawed, mixed, and heated to $37^{\circ} \mathrm{C}$ for $5 \mathrm{~min}$ and then centrifuged at $10,000 \mathrm{~g}$ for $5 \mathrm{~min}$. The supernatant was used for the analysis. Samples from patients and healthy control subjects were plated on the assay plate in a randomly assigned sequence. $\left[{ }^{15} \mathrm{~N}_{2}{ }^{13} \mathrm{C}_{1}\right]$ 8-oxoGuo, the stable isotope labeled 8-oxoGuo, was customized from Toronto Research Chemicals (Canada). 8 -oxodGuo ( $>98 \%$ purity) was purchased from SigmaAldrich (USA). 8-oxoGuo (>98\% purity) was purchased from ALEXIS Biochemicals (USA). The urinary content of the oxidized nucleosides 8-oxodGuo and 8-oxoGuo was quantified using a modified ultraperformance liquid chromatography and mass spectrometry (UPLC-MS/MS) assay established by our laboratory. Briefly, the chromatographic separation was performed on an Acquity UPLC system (Waters Corporation, Milford, USA) using an Acquity UPLC BEH C18 column $(1.7 \mu \mathrm{m}, 2.1 \times 50 \mathrm{~mm}$; Waters Corp. $)$ and a VanGuard precolumn $(1.7 \mu \mathrm{m}, 2.1 \times 5 \mathrm{~mm}$; Waters Corp. $)$ with a column temperature of $25^{\circ} \mathrm{C}$. A waters ACQUITY UPLC equipped with an Xevo TQ-S triple quadrupole mass spectrometer was used for measurement.

The mobile phase contained $0.1 \%(\mathrm{v} / \mathrm{v})$ formic acid (A) and 100\% methanol (B); gradient elution was applied to obtain the best peak shape (see Supplementary Table 1 in Supplementary Material available online at http://dx.doi .org/10.1155/2016/4323198). Electrospray ionization was performed in the positive ion mode. The multiple reaction monitoring (MRM) mode was applied during quantification. The desolvation temperature was set at $500^{\circ} \mathrm{C}$. Capillary and cone voltages were set at $2.5 \mathrm{KV}$ and $20 \mathrm{~V}$. Quantification was based on the signal peak area from transitions $\mathrm{m} / \mathrm{z}$ $283.9 \rightarrow 167.9$ (8-oxodGuo) and $299.9 \rightarrow 167.9$ (8-oxoGuo) related to the peak area of the $\left[{ }^{15} \mathrm{~N}_{2}{ }^{13} \mathrm{C}_{1}\right] 8$-oxoGuo $\mathrm{m} / z$ $302.9 \rightarrow 170.9$. 8-oxodGuo and 8-oxoGuo were eluted at 1.63 and 1.32 minutes, respectively. Considering the variability among the urinary volumes and the significant differences in the renal glomerular function, urinary levels of 8-oxodGuo and 8-oxoGuo were normalized to the urinary creatinine concentration. Laboratory personnel performing the analysis were blinded to the category of participants and the clinical state of patients with type 2 diabetes.

2.4. Statistical Analysis. The clinical characteristics of the participants were expressed as mean \pm SD for normally distributed variables and were compared between groups by independent $t$-test. The chi-square test was used to determine the significance of the differences in the distribution of categorical data. Pearson's correlation analysis was performed to examine the relationships between nucleotide acid oxidation markers and factors of interest. Ordinal regression analysis was further used to determine the relationships between nucleotide acid oxidation markers and the variables. For all parametric tests, statistical analyses were performed using SPSS 21.0 software (IBM Corporation, New York, NY, USA) and were plotted with GraphPad Prism 5.0 software (GraphPad Software Inc., La Jolla, CA, USA). Additional information on statistical methods is available in the Supplementary Material. A two-sided $p$ value $<0.05$ was deemed statistically significant.

\section{Results}

3.1. Demographic and Clinical Characteristics. Demographic and clinical characteristics of the study subjects are described in Table 1. All of the study participants were Chinese and the patients received specialized treatment at the Endocrinology Department of West China Hospital. There were no significant differences in age and gender between healthy controls and diabetes patients. Glucose levels were significantly higher in patients with type 2 diabetes in contrast with the healthy 
TABLE 1: Demographic and clinical characteristics of the study participants.

\begin{tabular}{|c|c|c|c|c|c|}
\hline \multirow[b]{2}{*}{ Variables } & \multirow[b]{2}{*}{$\begin{array}{l}\text { Healthy controls } \\
\qquad(n=683)\end{array}$} & \multicolumn{3}{|c|}{ T2DM } & \multirow[b]{2}{*}{$p$} \\
\hline & & $\begin{array}{c}\text { All } \\
(n=633)\end{array}$ & $\begin{array}{l}\text { Without complications } \\
\qquad(n=366)\end{array}$ & $\begin{array}{l}\text { With complications } \\
\qquad(n=267)\end{array}$ & \\
\hline Age, years, mean $\pm \mathrm{SD}^{\dagger}$ & $57.1 \pm 12.6$ & $57.2 \pm 12.4$ & $57.6 \pm 11.7$ & $58.8 \pm 11.6$ & $0.12^{*} / 0.176^{* *}$ \\
\hline Gender (male/female) $)^{\ddagger}$ & $362 / 321$ & $364 / 269$ & $217 / 149$ & $147 / 120$ & $0.11^{*} / 0.288^{* *}$ \\
\hline HbAlc (\%) & - & $7.90 \pm 3.51$ & $8.00 \pm 4.27$ & $7.76 \pm 1.93$ & $0.129^{* *}$ \\
\hline Fasting blood glucose $(\mathrm{mmol} / \mathrm{L})^{\dagger}$ & $5.44 \pm 0.75$ & $8.68 \pm 3.14$ & $8.90 \pm 3.22$ & $8.38 \pm 3.02$ & $<0.001^{*} / 0.03^{* *}$ \\
\hline Triglyceride $(\mathrm{mmol} / \mathrm{L})^{\dagger}$ & $1.41 \pm 0.94$ & $1.64 \pm 1.07$ & $1.67 \pm 1.08$ & $1.59 \pm 1.06$ & $<0.001^{*} / 0.47^{* *}$ \\
\hline Total cholesterol $(\mathrm{mmol} / \mathrm{L})^{\dagger}$ & $4.95 \pm 0.96$ & $4.59 \pm 1.10$ & $4.60 \pm 1.00$ & $4.58 \pm 1.23$ & $<0.001^{*} / 0.48^{* *}$ \\
\hline HDL cholesterol $(\mathrm{mmol} / \mathrm{L})^{\dagger}$ & $1.58 \pm 0.44$ & $1.38 \pm 0.44$ & $1.35 \pm 0.38$ & $1.41 \pm 0.50$ & $<0.001^{*} / 0.36^{* *}$ \\
\hline $\mathrm{LDL}$ cholesterol $(\mathrm{mmol} / \mathrm{L})^{\dagger}$ & $2.91 \pm 0.75$ & $2.67 \pm 0.94$ & $2.67 \pm 0.84$ & $2.66 \pm 1.07$ & $<0.001^{*} / 0.38^{* *}$ \\
\hline
\end{tabular}

Data expressed as arithmetic mean \pm standard deviation or $n$.

${ }^{\dagger}$ Statistical differences between groups tested using unpaired Student's $t$ test. ${ }^{*}$ Statistical differences between groups tested using chi-square test. ${ }^{*}$ Statistical differences between healthy controls and patients with T2DM. ${ }^{* *}$ Statistical differences between T2DM without complications and T2DM with complications.

TABLE 2: Urinary levels of 8-oxodGuo and 8-oxoGuo in the different age group.

\begin{tabular}{|c|c|c|c|c|c|c|}
\hline & \multicolumn{6}{|c|}{ Age group } \\
\hline & $31-40$ (years) & $41-50$ (years) & $51-60$ (years) & 61-70 (years) & $71-80$ (years) & All $\{31-80$ (years) $\}$ \\
\hline \multicolumn{7}{|l|}{ 8-oxodGuo ( $\mu \mathrm{mol} / \mathrm{mol}$ creatinine $)$} \\
\hline Healthy controls & $1.27 \pm 0.58$ & $1.25 \pm 0.56$ & $1.73 \pm 0.76$ & $2.02 \pm 0.95$ & $2.02 \pm 1.04$ & $1.68 \pm 0.87$ \\
\hline Diabetes without complications & $1.48 \pm 0.62$ & $1.65 \pm 0.86$ & $1.80 \pm 1.01$ & $1.80 \pm 1.04$ & $1.84 \pm 1.07$ & $1.75 \pm 0.97$ \\
\hline Diabetes with complications & $1.53 \pm 0.96$ & $1.67 \pm 1.00$ & $1.84 \pm 0.98$ & $1.62 \pm 0.97$ & $1.75 \pm 0.81$ & $1.70 \pm 0.95$ \\
\hline \multicolumn{7}{|l|}{ 8-oxoGuo ( $\mu \mathrm{mol} / \mathrm{mol}$ creatinine) } \\
\hline Healthy controls & $1.65 \pm 0.47$ & $1.81 \pm 0.55$ & $2.22 \pm 0.72$ & $2.58 \pm 0.88$ & $2.82 \pm 0.86$ & $2.25 \pm 0.84$ \\
\hline Diabetes without complications & $2.09 \pm 0.78$ & $2.19 \pm 0.97$ & $2.64 \pm 1.04$ & $2.63 \pm 1.08$ & $3.07 \pm 1.29$ & $2.55 \pm 1.10$ \\
\hline Diabetes with complications & $2.45 \pm 1.67$ & $2.38 \pm 1.01$ & $3.01 \pm 1.32$ & $2.70 \pm 1.17$ & $3.34 \pm 1.24$ & $2.81 \pm 1.27$ \\
\hline
\end{tabular}

controls $(p<0.001)$. There was no significant difference between diabetes patients with complications and those without complications in HbAlc $(7.76 \pm 1.93$ versus $8.00 \pm$ $4.27 \%, p=0.129$ ). Blood lipid levels such as triglyceride, total cholesterol, HDL, and LDL cholesterol also had no significant differences between diabetes patients with complications and those without complications.

3.2. Higher Levels of 8-oxodGuo and 8-oxoGuo in Diabetes Patients Than in Healthy Controls. An UPLC-MS/MS chromatogram of $\left[{ }^{15} \mathrm{~N}_{2}{ }^{13} \mathrm{C}_{1}\right]$ 8-oxoGuo, 8-oxoGuo, and 8oxodGuo is shown in Supplementary Figure 1A together with a chromatogram of a urine sample (Supplementary Figure 1B). The urinary levels of 8-oxodGuo and 8-oxoGuo in healthy controls and diabetes patients are showed in Table 2. We noted that age played an important role in both the DNA marker 8-oxodGuo and the RNA marker 8-oxoGuo. Therefore, we subdivided all the participants into different groups with each group every 10 years old and further analyzed them in different age groups (Table 2 and Figure 1). In healthy controls, both 8-oxodGuo and 8-oxoGuo increased with age. The correlation coefficient of urinary 8-oxoGuo with age was 0.50 , which indicated a stronger degree of linear relationship than that of 8-oxodGuo with age $(r=0.35$; $p<0.001 ; n=683$ ) (Supplementary Figure 2).
Correlations between nucleic acid oxidation markers and variables of interest are presented in Table 3. In order to assess the effects of different variables on 8-oxodGuo and 8oxoGuo, we further performed ordinal regression analysis (Supplementary Table 2). The results also showed that age played an important part in nucleic acid oxidation of both 8-oxodGuo and 8-oxoGuo. Moreover, we compared the correlations between oxidative stress markers and HbAlc in adults with type 2 diabetes. There was a moderate correlation between 8-oxoGuo and HbAlc $(r=0.16$; $p<0.001)$ (Supplementary Figure $3 \mathrm{~B})$. However, the correlation between 8 oxodGuo and $\mathrm{HbAlc}$ was not significant $(r=0.07 ; p>0.05)$ (Supplementary Figure 3A). In addition, the levels of urinary 8-oxodGuo and 8-oxoGuo were significantly correlated $(r=$ 0.642; $p<0.001 ; n=1,316$ ) (Supplementary Figure 4).

In all the participants, the levels of both 8-oxodGuo and 8-oxoGuo were significantly higher in diabetes with/without complications group compared with the age-matched control group ( $p=0.02$ and $p<0.001$, resp.). Furthermore, we compared the changes of 8-oxodGuo and 8-oxoGuo in the patients with complications and those without complications. For 8-oxodGuo, it was slightly higher in diabetes with complications compared to those without complications with age between 31 and 60 years (Figure 1(a)). For 8-oxoGuo, there was a rather clear and significant increasing trend 
TABLE 3: Pearson's correlation coefficients of nucleic acid oxidation with variables of interest.

\begin{tabular}{|c|c|c|c|c|c|c|}
\hline \multirow{2}{*}{ Study subjects } & \multicolumn{2}{|c|}{ All } & \multicolumn{2}{|c|}{ Healthy controls } & \multicolumn{2}{|c|}{ Type 2 diabetes } \\
\hline & $r$ & $p$ & $r$ & $p$ & $r$ & $p$ \\
\hline \multicolumn{7}{|l|}{ 8-oxodGuo } \\
\hline Age & 0.208 & $<0.001$ & 0.346 & $<0.001$ & 0.062 & 0.120 \\
\hline Fasting blood glucose & 0.019 & 0.509 & 0.074 & 0.063 & 0.004 & 0.919 \\
\hline Triglyceride & -0.042 & 0.140 & -0.055 & 0.167 & -0.038 & 0.365 \\
\hline Total cholesterol & -0.025 & 0.381 & 0.060 & 0.127 & -0.092 & 0.028 \\
\hline HDL cholesterol & 0.041 & 0.150 & 0.125 & 0.002 & -0.029 & 0.494 \\
\hline LDL cholesterol & -0.057 & 0.048 & 0.015 & 0.709 & -0.108 & 0.010 \\
\hline \multicolumn{7}{|l|}{ 8-oxoGuo } \\
\hline Age & 0.350 & $<0.001$ & 0.497 & $<0.001$ & 0.239 & $<0.001$ \\
\hline Fasting blood glucose & 0.135 & $<0.001$ & 0.095 & 0.016 & 0.019 & 0.649 \\
\hline Triglyceride & 0.000 & 0.990 & 0.030 & 0.442 & -0.059 & 0.159 \\
\hline Total cholesterol & -0.037 & 0.199 & 0.099 & 0.012 & -0.074 & 0.078 \\
\hline HDL cholesterol & 0.009 & 0.750 & 0.122 & 0.002 & 0.002 & 0.953 \\
\hline LDL cholesterol & -0.073 & 0.011 & 0.007 & 0.854 & -0.081 & 0.055 \\
\hline
\end{tabular}

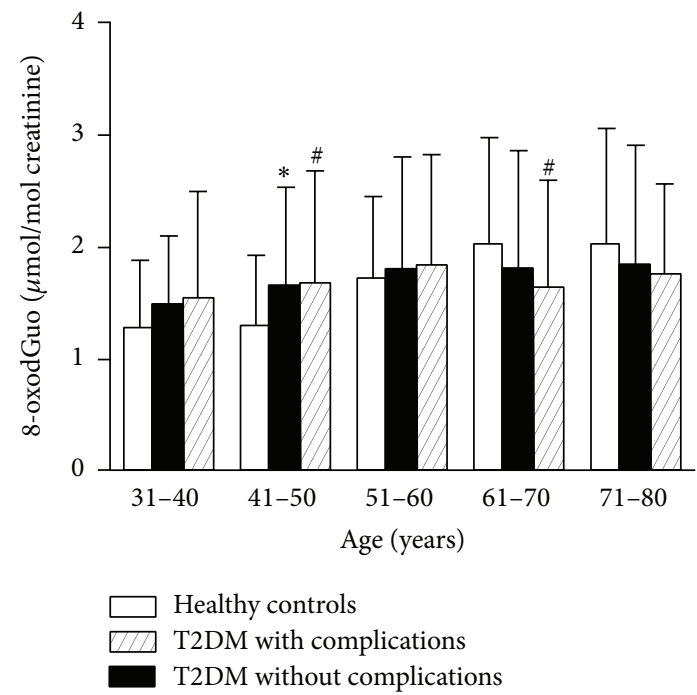

(a)

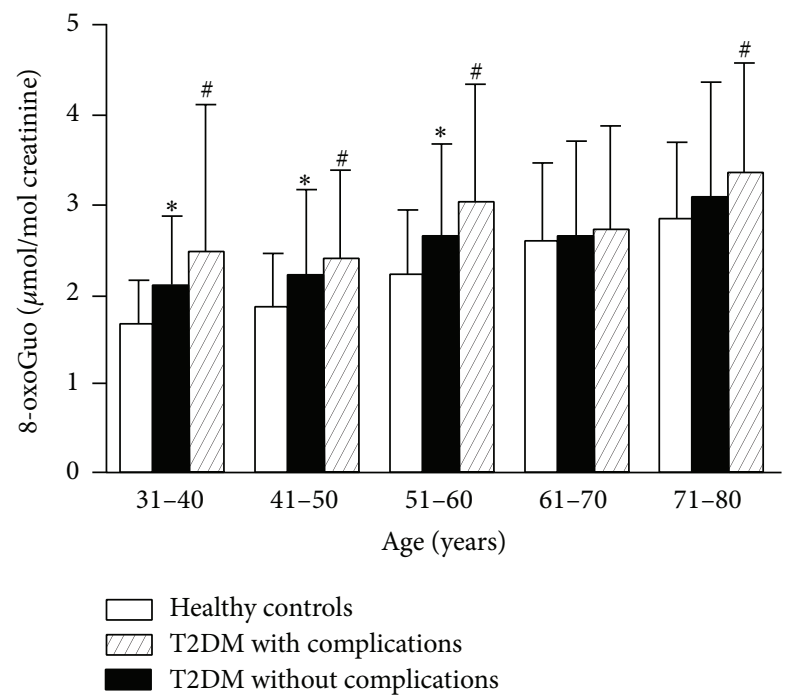

(b)

FIGURE 1: Urinary levels of 8-oxodGuo or 8-oxoGuo with age in healthy controls and patients. (a) Urinary 8-oxodGuo ( $\mu \mathrm{mol} / \mathrm{mol} \mathrm{creatinine}$ ) levels in healthy controls and patients with type 2 diabetes. (b) Urinary 8-oxoGuo ( $\mu \mathrm{mol} / \mathrm{mol}$ creatinine) levels in healthy controls and patients with type 2 diabetes. Columns represent mean \pm SD. ${ }^{*} p<0.05$ (statistical differences between healthy controls and diabetes patients without complications by Student's $t$-test). ${ }^{\#} p<0.05$ (statistical differences between T2DM with complications and diabetes patients without complications by Student's $t$-test). T2DM: type 2 diabetes mellitus.

in diabetes with/without complications compared to agematched healthy controls (Figure 1(b)). This increasing trend was seen in all the different age sets. And most pronounced difference was seen between diabetes patients with complications and those without complications ( $3.01 \pm 1.32$ versus $2.64 \pm 1.04 \mu \mathrm{mol} / \mathrm{mol}$ creatinine, $p<0.05)$ with age between 51 and 60 years (Table 2). And also we found that the levels of urinary 8-oxoGuo were obviously higher than 8-oxodGuo in the same age group of participants.

3.3. Levels of 8-oxoGuo in Diabetes Patients with Different Complications. There are many different types in diabetic complications, for example, microvascular complications and macrovascular complications. Considering that 8-oxoGuo had a more obvious increasing trend in diabetes complications than 8-oxodGuo, we analyzed the change of 8-oxoGuo levels in all the participants in Figure 2. Apparently, there was a significant difference in 8-oxoGuo between diabetes with macrovascular complications and those without complications $(3.21 \pm 1.36$ versus $2.53 \pm 1.05 \mu \mathrm{mol} / \mathrm{mol}$ creatinine, $p<0.001)$. And patients with diabetic nephropathy had higher levels of 8-oxoGuo than those without complications, but not significant. 


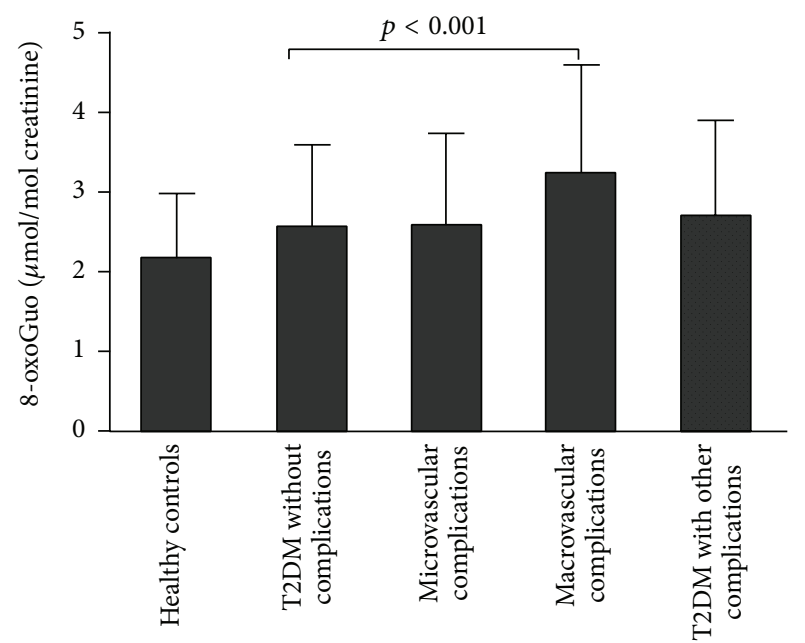

FIGURE 2: Urinary levels of 8-oxoGuo in diabetes patients with different complications. $p<0.01$ (statistical differences between patients with macrovascular complications and patients without complications by Student's $t$-test).

\section{Discussion}

With the increase of the diabetes incidence rate, more studies are needed to do research on its pathogenic mechanism and find the relevant therapies. Although the mechanisms underlying development of the disease are complex and varied, oxidative stress provides a new sight. We noted that many variables such as nucleic acid oxidation, serum glucose, and lipids had significant differences between diabetes patients and healthy controls, which was in accordance with some previous studies [15-17]. However, there were only 8oxoGuo, but not serum glucose, and lipids that had significant differences between diabetes patients with complications and those without complications. We also found that age had a significant effect on both 8-oxodGuo and 8-oxoGuo in the study subjects. This confirmed our previous research $[18,19]$ and also the review by Jacob et al. [20].

In combination with the previously published results, all these results suggest that urinary 8-oxodGuo and 8-oxoGuo are potentially useful biomarkers for evaluating the severity of complications in patients with type 2 diabetes. Compared with DNA, RNA is more prone to oxidative damage because of its widespread distribution in close proximity to sites of reactive oxygen species generation, its single stranded nature, unidentified active repair mechanism, and the lesser association with protecting proteins [21]. However, the focus on nucleic acid oxidation research has been centered on DNA $[22,23]$. We found that there was a clear and significant trend for increased risk in 8-oxoGuo between diabetes patients with complications compared to those without complications. However, the trend for 8-oxodGuo is moderate, which was consistent with previous studies [22, 24-27]. Further studies are needed to establish the RNA oxidation.

Some previous studies indicated that increased oxidative stress might have a primary role in the pathogenesis of diabetic complications, especially patients with nephropathy and vascular complications [22, 23, 28-31]. We also found that there was a significant difference in 8-oxoGuo between patients with macrovascular complications and those without complications. Broedbaek et al. [12] showed that urinary excretion of the RNA oxidation marker 8-oxoGuo independently predicted all-cause and diabetes-related mortality, whereas the DNA oxidation marker 8-oxodGuo did not. Therefore, urinary 8-oxoGuo could be a useful biomarker for diabetes with complications, especially in macrovascular complications.

It has been well known that cells have protective mechanisms against oxidative damage. However, oxidative stress occurs when the reactive oxygen species (ROS) level overwhelms defensive mechanisms, causing a cellular imbalance between pro- and antioxidant factors. Elevated levels of oxidation markers such as 8-oxodGuo and 8-oxoGuo probably lead to strand breaks and to oxidative base modifications; and multiple signaling pathways may also contribute to the adverse effects of glucotoxicity on cellular functions $[8,11]$. Therefore, higher levels of 8-oxodGuo and 8-oxoGuo indicate diabetic complications. In this study, the findings not only provide further insights into the pathophysiological mechanisms responsible for the progression of complications in diabetes but also indicate that 8-oxoGuo has more advantages than 8-oxodGuo in clinical applications.

The main strengths of our study are as follows. First, our study employed UPLC-MS/MS for the measurement of DNA and RNA oxidation, a method that was more specific and reliable than the enzyme-linked immunosorbent assay (ELISA) in urinary samples [32-34]. Next, we investigated the variation between the two measurements of 8-oxodGuo and 8-oxoGuo one week apart in healthy individuals and found a variability of less than $10 \%$ in the preliminary experiment, similarly reported by Barregard et al. [33]. In addition, we tested 8-oxodGuo and 8-oxoGuo in the urine rather than in blood, which was mainly explained by the following three reasons. Firstly, blood levels of 8-oxodGuo and 8oxoGuo were mainly determined by the kidney function and when different individuals were compared, they were thus unlikely to provide specific information about oxidative stress [24]. Secondly, urine contained a higher level of oxidized guanosine than blood, thus providing a higher accuracy. Thirdly, the sample of urine was obtained more easily and noninvasively than blood.

There are also some limitations in this study. First, consumption of medicines in diabetes patients was little known. Some studies reported that interventions such as antioxidants could decrease oxidative stress [35-37]. Second, glucose variability, which has been shown to exhibit a more specific triggering effect on oxidative stress than chronic sustained hyperglycemia [38-40], was not assessed in the study.

In summary, the present study demonstrated significantly higher levels of oxidatively generated damage to DNA and RNA in patients with type 2 diabetes compared with healthy control subjects. We suggest the role for 8-oxodGuo and 8-oxoGuo as a molecular mechanism contributing to the complications of type 2 diabetes. Nucleic acid oxidation, especially RNA oxidation, is a possible pathogenesis of type 
2 diabetes complications. Further studies should be initiated to evaluate the potential clinical applications of 8-oxoGuo as a biomarker in diabetes that could be used for risk stratification, progressive course, selection of appropriate therapeutic intervention, and the monitoring of response to therapy.

\section{Conflict of Interests}

The authors declare that there is no conflict of interests regarding the publication of this paper.

\section{Authors' Contribution}

Xinle Liu and Wei Gan contributed equally to this work.

\section{Acknowledgments}

The authors thank Shuang Wang and Tingting Li for their help in the collecting of the samples. This study was supported by the National Natural Science Foundation of China (no. 81401716).

\section{References}

[1] P. Z. Zimmet, D. J. Magliano, W. H. Herman, and J. E. Shaw, "Diabetes: A 21st century challenge," The Lancet Diabetes \& Endocrinology, vol. 2, no. 1, pp. 56-64, 2014.

[2] "Diagnosis and classification of diabetes mellitus," Diabetes Care, vol. 33, supplement 1, pp. S62-S69, 2009.

[3] T. Mazzone, A. Chait, and J. Plutzky, "Cardiovascular disease risk in type 2 diabetes mellitus: insights from mechanistic studies," The Lancet, vol. 371, no. 9626, pp. 1800-1809, 2008.

[4] A. Matheson, M. D. P. Willcox, J. Flanagan, and B. J. Walsh, "Urinary biomarkers involved in type 2 diabetes: a review," Diabetes/Metabolism Research and Reviews, vol. 26, no. 3, pp. 150-171, 2010.

[5] L. Piconi, L. Quagliaro, and A. Ceriello, "Oxidative stress in diabetes," Clinical Chemistry and Laboratory Medicine, vol. 41, no. 9, pp. 1144-1149, 2003.

[6] A. C. Maritim, R. A. Sanders, and J. B. Watkins III, "Diabetes, oxidative stress, and antioxidants: a review," Journal of Biochemical and Molecular Toxicology, vol. 17, no. 1, pp. 24-38, 2003.

[7] J. L. Evans, I. D. Goldfine, B. A. Maddux, and G. M. Grodsky, "Oxidative stress and stress-activated signaling pathways: a unifying hypothesis of type 2 diabetes," Endocrine Reviews, vol. 23, no. 5, pp. 599-622, 2002.

[8] L. Rochette, M. Zeller, Y. Cottin, and C. Vergely, "Diabetes, oxidative stress and therapeutic strategies," Biochimica et Biophysica Acta-General Subjects, vol. 1840, no. 9, pp. 2709-2729, 2014.

[9] M. Dizdaroglu, P. Jaruga, M. Birincioglu, and H. Rodriguez, "Free radical-induced damage to DNA: mechanisms and measurement," Free Radical Biology and Medicine, vol. 32, no. 11, pp. 1102-1115, 2002.

[10] A. Weimann, D. Belling, and H. E. Poulsen, "Quantification of 8-oxo-guanine and guanine as the nucleobase, nucleoside and deoxynucleoside forms in human urine by high-performance liquid chromatography-electrospray tandem mass spectrometry," Nucleic Acids Research, vol. 30, no. 2, article E7, 2002.
[11] H. E. Poulsen, E. Specht, K. Broedbaek et al., "RNA modifications by oxidation: a novel disease mechanism?" Free Radical Biology \& Medicine, vol. 52, no. 8, pp. 1353-1361, 2012.

[12] K. Broedbaek, V. Siersma, T. Henriksen et al., "Association between urinary markers of nucleic acid oxidation and mortality in type 2 diabetes: a population-based cohort study," Diabetes Care, vol. 36, no. 3, pp. 669-676, 2013.

[13] K. Broedbaek, V. Siersma, T. Henriksen et al., "Urinary markers of nucleic acid oxidation and cancer in type 2 diabetes," Redox Biology, vol. 4, pp. 34-39, 2015.

[14] K. G. M. M. Alberti and P. Z. Zimmet, "Definition, diagnosis and classification of diabetes mellitus and its complications. Part 1: diagnosis and classification of diabetes mellitus provisional report of a WHO consultation," Diabetic Medicine, vol. 15, no. 7, pp. 539-553, 1998.

[15] R. M. Krauss, "Lipids and lipoproteins in patients with type 2 diabetes," Diabetes Care, vol. 27, no. 6, pp. 1496-1504, 2004.

[16] M. Kanauchi, H. Nishioka, and T. Hashimoto, "Oxidative DNA damage and tubulointerstitial injury in diabetic nephropathy," Nephron, vol. 91, no. 2, pp. 327-329, 2002.

[17] P. Dandona, K. Thusu, S. Cook et al., "Oxidative damage to DNA in diabetes mellitus," The Lancet, vol. 347, no. 8999, pp. 444-445, 1996.

[18] W. Gan, B. Nie, F. Shi et al., "Age-dependent increases in the oxidative damage of DNA, RNA, and their metabolites in normal and senescence-accelerated mice analyzed by LCMS/MS: urinary 8-oxoguanosine as a novel biomarker of aging," Free Radical Biology \& Medicine, vol. 52, no. 9, pp. 1700-1707, 2012.

[19] W.-X. Wang, S.-B. Luo, M.-M. Xia et al., "Analysis of the oxidative damage of DNA, RNA, and their metabolites induced by hyperglycemia and related nephropathy in Sprague Dawley rats," Free Radical Research, vol. 49, no. 10, pp. 1199-1209, 2015.

[20] K. D. Jacob, N. Noren Hooten, A. R. Trzeciak, and M. K. Evans, "Markers of oxidant stress that are clinically relevant in aging and age-related disease," Mechanisms of Ageing and Development, vol. 134, no. 3-4, pp. 139-157, 2013.

[21] B. Nie, W. Gan, F. Shi et al., "Age-dependent accumulation of 8-oxoguanine in the DNA and RNA in various rat tissues," Oxidative Medicine and Cellular Longevity, vol. 2013, Article ID 303181, 9 pages, 2013.

[22] H.-Z. Pan, L. Zhang, M.-Y. Guo et al., "The oxidative stress status in diabetes mellitus and diabetic nephropathy," Acta Diabetologica, vol. 47, no. 1, pp. S71-S76, 2010.

[23] Y. Hinokio, S. Suzuki, M. Hirai, C. Suzuki, M. Suzuki, and T. Toyota, "Urinary excretion of 8-oxo-7, 8-dihydro-2' deoxyguanosine as a predictor of the development of diabetic nephropathy," Diabetologia, vol. 45, no. 6, pp. 877-882, 2002.

[24] H. E. Poulsen, L. L. Nadal, K. Broedbaek, P. E. Nielsen, and A. Weimann, "Detection and interpretation of 8-oxodG and 8oxoGua in urine, plasma and cerebrospinal fluid," Biochimica et Biophysica Acta, vol. 1840, no. 2, pp. 801-808, 2014.

[25] W. Martinet, G. R. Y. de Meyer, A. G. Herman, and M. M. Kockx, "RNA damage in human atherosclerosis: pathophysiological significance and implications for gene expression studies," RNA Biology, vol. 2, no. 1, pp. 4-7, 2005.

[26] Q. Kong and C.-L. G. Lin, "Oxidative damage to RNA: mechanisms, consequences, and diseases," Cellular and Molecular Life Sciences, vol. 67, no. 11, pp. 1817-1829, 2010.

[27] W. I. Sivitz and M. A. Yorek, "Mitochondrial dysfunction in diabetes: from molecular mechanisms to functional significance 
and therapeutic opportunities," Antioxidants \& Redox Signaling, vol. 12, no. 4, pp. 537-577, 2010.

[28] O. Tabak, R. Gelisgen, H. Erman et al., "Oxidative lipid, protein, and DNA damage as oxidative stress markers in vascular complications of diabetes mellitus," Clinical and Investigative Medicine, vol. 34, no. 3, pp. E163-E171, 2011.

[29] P. J. Beisswenger, "Glycation and biomarkers of vascular complications of diabetes," Amino Acids, vol. 42, no. 4, pp. 1171-1183, 2012.

[30] D. Ziegler, S. Buchholz, C. Sohr, J. Nourooz-Zadeh, and M. Roden, "Oxidative stress predicts progression of peripheral and cardiac autonomic nerve dysfunction over 6 years in diabetic patients," Acta Diabetologica, vol. 52, no. 1, pp. 65-72, 2015.

[31] C.-C. Sung, Y.-C. Hsu, C.-C. Chen, Y.-F. Lin, and C.-C. $\mathrm{Wu}$, "Oxidative stress and nucleic acid oxidation in patients with chronic kidney disease," Oxidative Medicine and Cellular Longevity, vol. 2013, Article ID 301982, 15 pages, 2013.

[32] L. W. Garratt, V. Mistry, R. Singh et al., "Interpretation of urinary 8-oxo-7,8-dihydro- $2^{\prime}$-deoxyguanosine is adversely affected by methodological inaccuracies when using a commercial ELISA," Free Radical Biology and Medicine, vol. 48, no. 11, pp. 1460-1464, 2010.

[33] L. Barregard, P. Møller, T. Henriksen et al., "Human and methodological sources of variability in the measurement of urinary 8-oxo-7,8-dihydro-2' -deoxyguanosine," Antioxidants \& Redox Signaling, vol. 18, no. 18, pp. 2377-2391, 2013.

[34] T. Henriksen, P. R. Hillestrøm, H. E. Poulsen, and A. Weimann, "Automated method for the direct analysis of 8-oxo-guanosine and 8-oxo- $2^{\prime}$-deoxyguanosine in human urine using ultraperformance liquid chromatography and tandem mass spectrometry," Free Radical Biology \& Medicine, vol. 47, no. 5, pp. 629-635, 2009.

[35] P. E. Pergola, P. Raskin, R. D. Toto et al., "Bardoxolone methyl and kidney function in CKD with type 2 diabetes," The New England Journal of Medicine, vol. 365, no. 4, pp. 327-336, 2011.

[36] Y. Wang, S. Ye, Y. Hu, L. Zhao, and M. Zheng, "The effect of hydrochloride pioglitazone on urinary 8-hydroxy-deoxyguanosine excretion in type 2 diabetics," Journal of Diabetes and its Complications, vol. 27, no. 1, pp. 75-77, 2013.

[37] U. Schmid, H. Stopper, A. Heidland, and N. Schupp, "Benfotiamine exhibits direct antioxidative capacity and prevents induction of DNA damage in vitro," Diabetes/Metabolism Research and Reviews, vol. 24, no. 5, pp. 371-377, 2008.

[38] L. Monnier, E. Mas, C. Ginet et al., "Activation of oxidative stress by acute glucose fluctuations compared with sustained chronic hyperglycemia in patients with type 2 diabetes," Journal of the American Medical Association, vol. 295, no. 14, pp. 1681-1687, 2006.

[39] A. Ceriello, K. Esposito, L. Piconi et al., "Oscillating glucose is more deleterious to endothelial function and oxidative stress than mean glucose in normal and type 2 diabetic patients," Diabetes, vol. 57, no. 5, pp. 1349-1354, 2008.

[40] C.-M. Chang, C.-J. Hsieh, J.-C. Huang, and I.-C. Huang, "Acute and chronic fluctuations in blood glucose levels can increase oxidative stress in type 2 diabetes mellitus," Acta Diabetologica, vol. 49, no. 1, pp. S171-S177, 2012. 


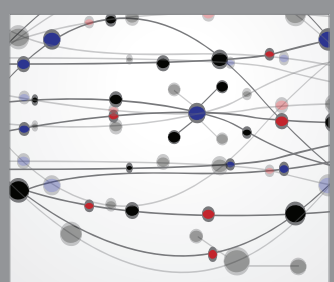

The Scientific World Journal
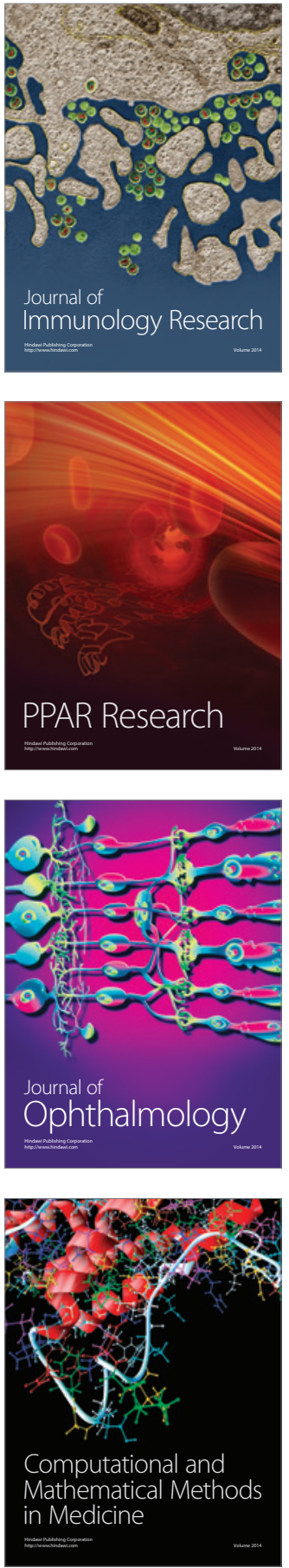

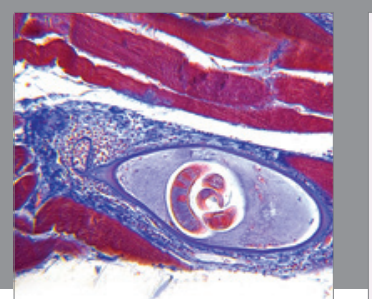

Gastroenterology Research and Practice

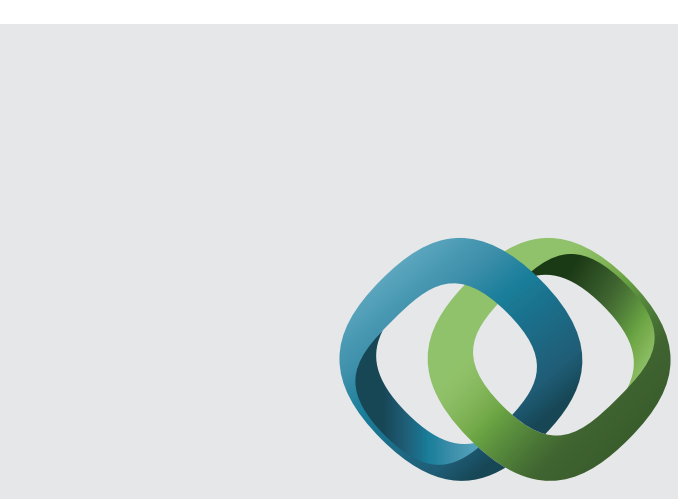

\section{Hindawi}

Submit your manuscripts at

http://www.hindawi.com
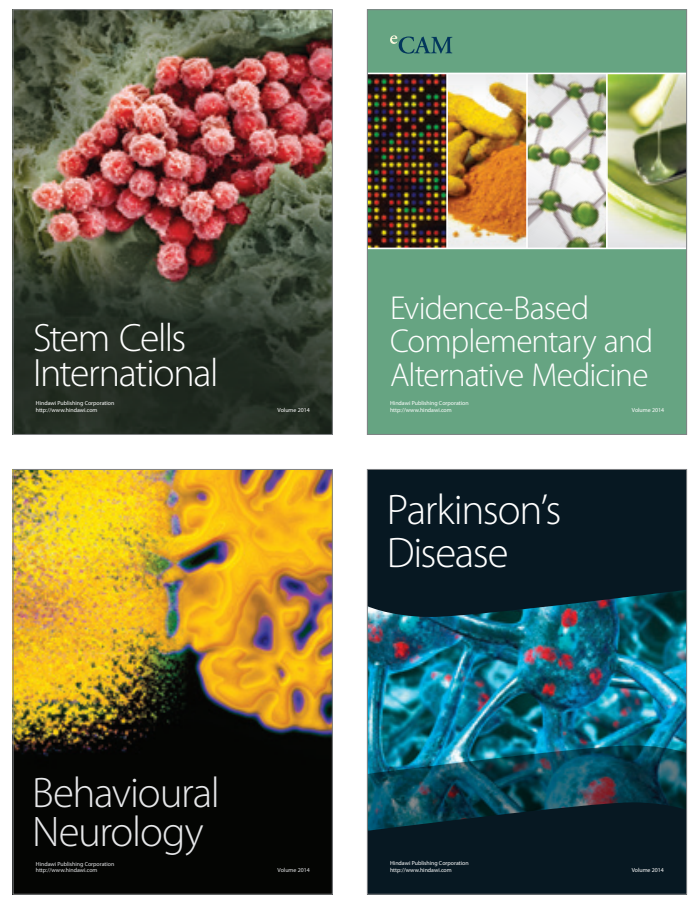
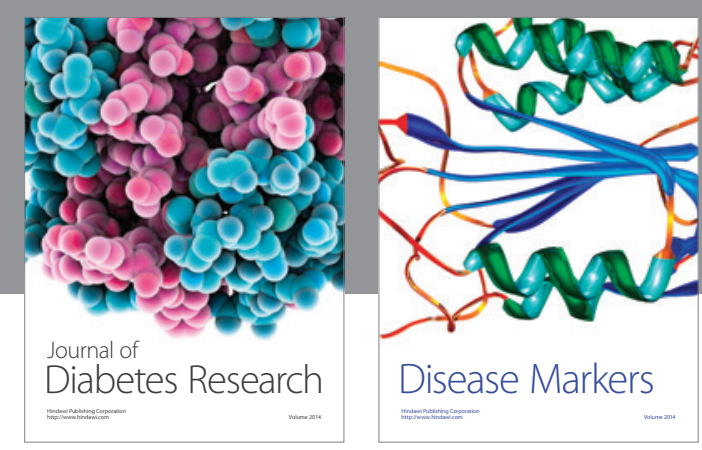

Disease Markers
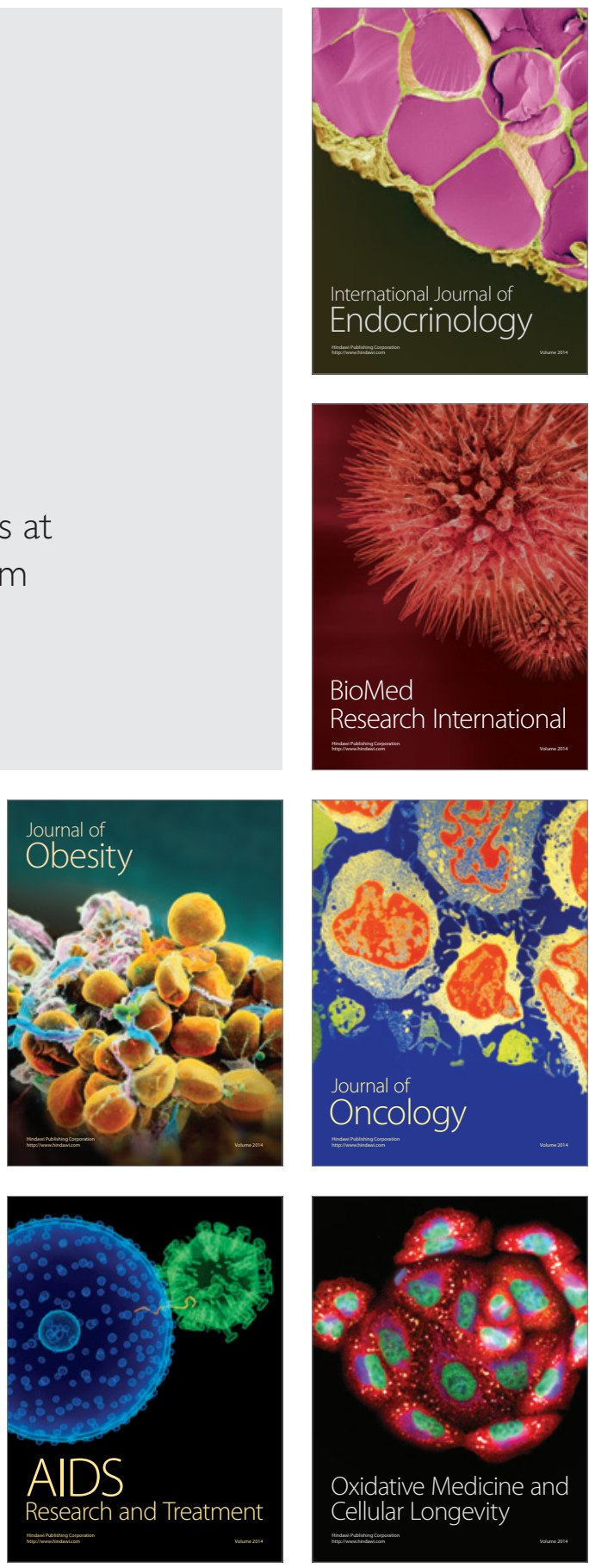